\title{
Stacking, Strain, \& Stiffness of 2D Transition Metal Dichalcogenides Quantified through Reciprocal Space
}

\author{
Noah Schnitzer ${ }^{1}$, Suk Hyun Sung ${ }^{1}$ and Robert Hovden ${ }^{1,2}$ \\ 1. Department of Materials Science and Engineering, University of Michigan, Ann Arbor, MI. \\ 2. Applied Physics Program, University of Michigan, Ann Arbor, MI.
}

Interest in 2D materials has expanded to multilayered systems where electronic, optical, and mechanical properties change - often dramatically - with stacking order, thickness, twist, and interlayer spacing $[1,2,3,4]$. Toward the fabrication of real $2 \mathrm{D}$ devices, atomic structure throughout all layers must be determined across billions of atoms that span micron length scales. Here we present selected area electron diffraction (SAED) techniques for fast identification of multilayer structure in all dimensions with subAngstrom precision, across many 2D materials. Moreover, we demonstrate the techniques' ability to quantitatively extract key structural parameters in 2D materials such as surface roughness, inter- \& intralayer spacings, and stacking order with methods available to anyone with access to a rudimentary TEM.

In reciprocal (k) space, 2D materials are structurally described by a set of rods directed perpendicular $\left(\mathrm{k}_{\mathrm{z}}\right)$ to the reciprocal hexagonal lattice (Fig. 1a, d). In the simplest case - a single layer of graphene (SLG) the intensity and phase of each rod is constant. Upon addition of a second layer (Fig. 1b), bilayer graphene (BLG) rods vary sinusoidally in both phase and intensity (Fig. 1a) with symmetry that uniquely defines each of the three registered stacking orders: AB/BA/AA (Fig. 1c). Structure in k-space becomes more complex for transition metal dichalcogenides (TMDs) due to the three atomic planes that comprise each layer (Fig 1e). Figure 1d shows inversion symmetric bilayer $2 \mathrm{H}-\mathrm{MoS}_{2}$ rods with intensity and phase that beat asymmetrically about the $\mathrm{k}_{\mathrm{z}}=0$ plane. Notably, the inversion symmetry is absent in single vdW layer $\mathrm{MoS}_{2}$ (Fig 1f); this broken symmetry is famously associated with the formation of a direct bandgap [1]. As the number of layers in a 2D material increases, the rod like structure gives way to discrete peaks along the $\mathrm{k}_{\mathrm{z}}$ direction (400 layers shown in Fig. 1f) as expected in bulk materials.

To experimentally probe the full 3D reciprocal space structure, SAED at many specimen tilts rocks the Ewald sphere and captures slices through k-space (Fig. 2c). This method reveals out-of-plane information invisible to conventional S/TEM (Fig. 2a, b). From our SAED tilt series on a single layer of suspended $2 \mathrm{H}-\mathrm{MoS}_{2}$, we report an intralayer chalcogen-chalcogen spacing $\left(\lambda_{\mathrm{ch}}\right)$ of $3.07 \pm 0.11 \AA$ (Fig. $\left.2 \mathrm{~d}\right)-$ comparable to the literature result of $3.01 \AA$ in the bulk [5]. Furthermore, rod broadening along $\mathrm{k}_{\mathrm{z}}$ is directly related to out-of-plane deformation. Measuring the rod divergence, we find the surface roughness of the monolayer $\mathrm{MoS}_{2}$ spans an angular range of $5.4^{\circ}$ corresponding to a strain of $0.45 \%$ - half of the previous result for SLG [3]. These measurements demonstrate the effectiveness of reciprocal space approaches for determination of principal structural parameters in 2D multilayered materials.

References:

[1] Splendiani, A. et al, Nano Letters 10(4) (2010), p. 1271.

[2] Xia, Ming et al, ACS Nano 9(12) (2015), p.12246.

[3] Meyer, J.C. et al, Nature 446(7131) (2007), p. 60.

[4] Kim, C.J. et al, Nano Letters 13(11) (2013), p. 5660.

[5] Schonfeld, B. et al, Acta Crystallographica 39 (1983), p. 404. 


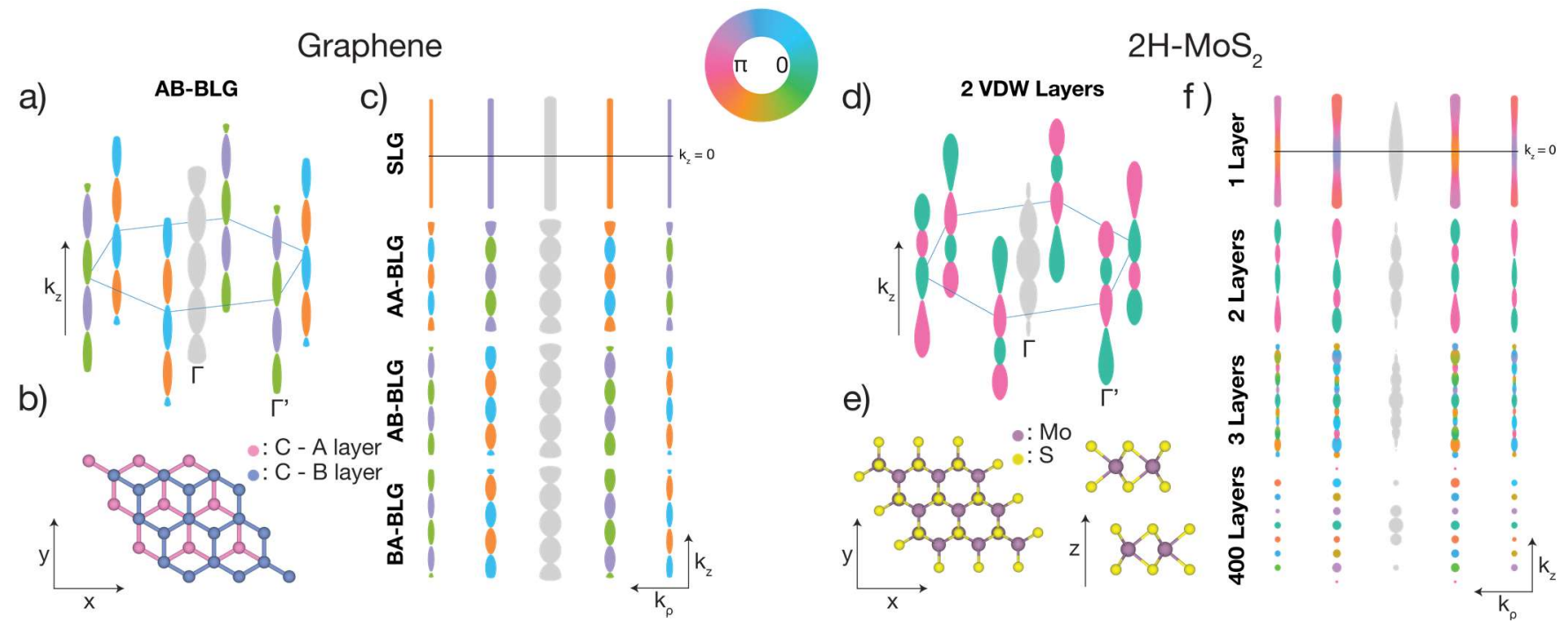

Figure 1. Kinematic Model of 3D reciprocal structure of 2D materials. Fully kinematic 3D reciprocal space structure of a) Bernal (AB) stacked bilayer graphene and d) $2 \mathrm{H}-\mathrm{MoS}_{2}$. Radius and color of the rods indicate the complex magnitude and phase, the hexagon marks $\mathrm{k}_{\mathrm{z}}=0$. Real space structure of $\left.\mathbf{b}\right) \mathrm{AB}-\mathrm{BLG}$ and e) $2 \mathrm{H}-\mathrm{MoS}_{2}$ are illustrated. The inversion symmetry of the two systems are shown both in real and reciprocal space. $\mathrm{k}_{\mathrm{z}}$ dependence of Bragg peaks of c) SLG, and AA, AB, BA stacked BLG and f) 1, 2, 3 and 400 layer $2 \mathrm{H}-\mathrm{MoS}_{2}$ are demonstrated. As number of atomic layers increases, the reciprocal rods become discrete.
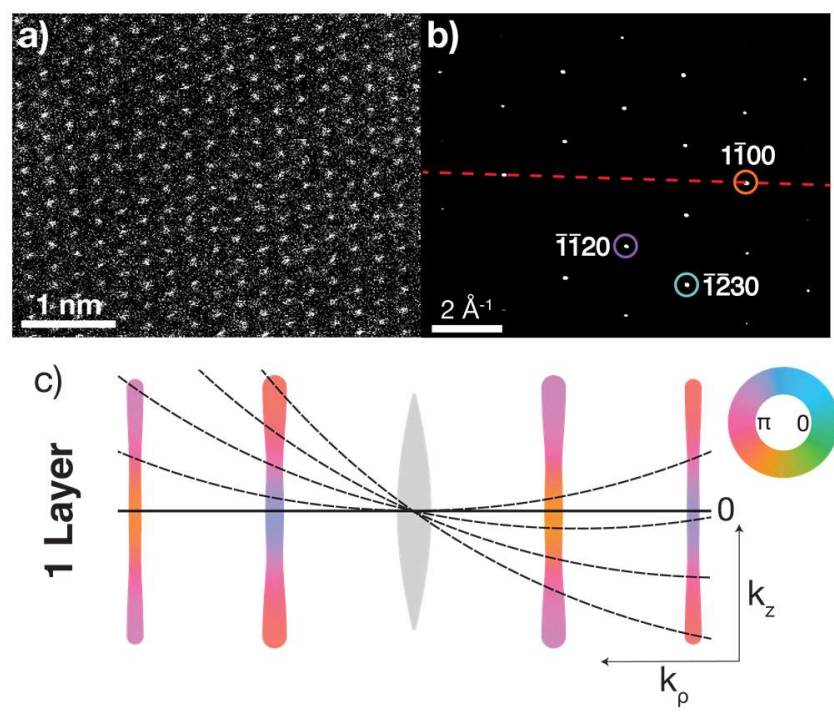

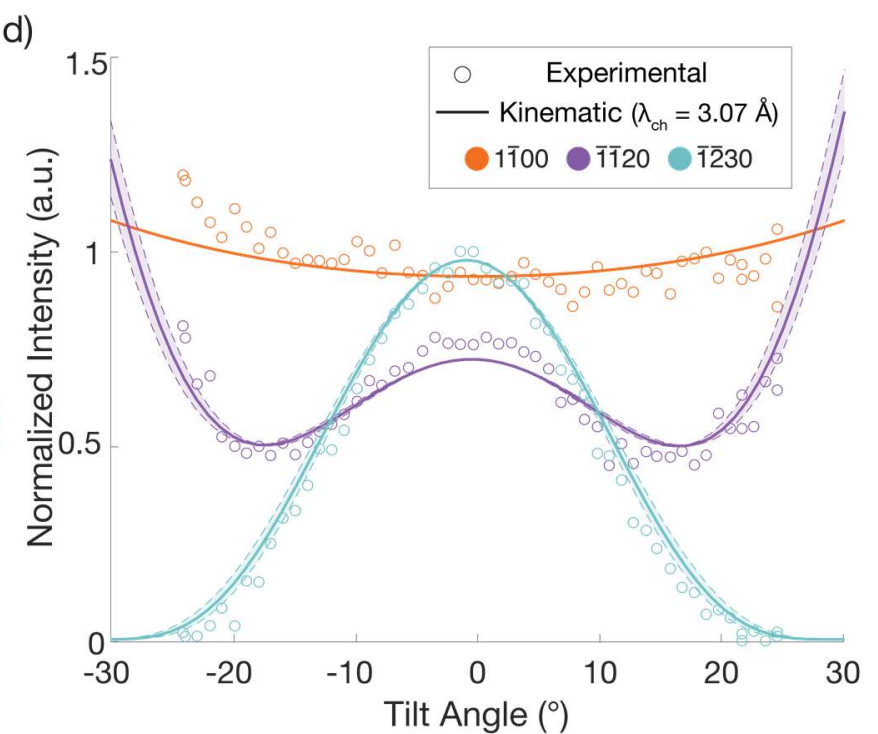

Figure 2. Experimentally probing the real and reciprocal space structure of single layer $\mathrm{MoS}_{2}$. A single $\mathrm{vdW}$ layer $2 \mathrm{H}-\mathrm{MoS}_{2}$ characterized both in a) real space by HAADF STEM and b) reciprocal space with SAED. Bright spots in a) represent individual Mo atoms. c) Diffraction tilt series probes $\mathrm{k}_{\mathrm{z}}$ space by tilting the Ewald sphere. The curvature of the Ewald sphere is exaggerated in the schematic. d) Integrated intensity of experimental and kinematically modeled diffraction spots indicated in b) plotted against tilt angle. Tilt axis is marked in b) as red dotted line. Inter-chalcogen spacing was determined to be $3.07 \pm$ $0.11 \AA$ by non-linear least squares fit of the experimental data to the kinematic model. $95 \%$ confidence interval for $\lambda_{\text {ch }}$ indicated by shaded regions. 